\title{
Research Paper: Relationship Between Self-Management Strategy and Self-Efficacy Among Staff of Ardabil Disaster and crossuark Emergency Medical Management Centers
}

\author{
Mohammad Taghi Amini ${ }^{1}$, Reza Noroozi ${ }^{1^{*}}$ \\ 1. Department of Management, Tehran Branch, Payame Noor University, Tehran, Iran.
}

ditation: Amini MT, Noroozi R. Relationship Between Self-Management Strategy and Self-Efficacy Among Staff of Ardabil Disaster and Emergency Medical Management Centers. Health in Emergencies and Disasters Quarterly. 2018; 3(2):85-90. https://doi.org/10.29252/nrip.hdq.3.2.85

https://doi.org/10.29252/nrip.hdq.3.2.85

Article info:

Received: 05 Jul. 2017

Accepted: 03 Nov. 2017

\section{Keywords:}

Job stress, Safety behavior, Self-efficacy, Self-management

\section{ABSTRACT}

Background: The nature of the work in the emergency department is as if the unwilling persons are in trouble. Self-efficacy is necessary to overcome obstacles in this type of job. Thus, the purpose of this study was to determine the relationship between self-management strategy and self-efficacy of emergency staff, stressing on the mediating role of job stress and safety behavior.

Materials and Methods: The present study is a non-experimental design and more precisely a correlation scheme of structural equation type. The study population consisted of all emergency department staff, including road and city campus in Ardabil province in 2017. To select the study sample group, 5 personnel from 40 bases in the entire province were randomly selected. A total of 200 employees were selected as the sample of the study. Harris job Stress Questionnaire (1995), General Health Questionnaire, Safety Behavior Questionnaire, and Self-management Strategy Questionnaire were used for data collection.

Results: The results showed a significant negative relationship between job stress and selfefficacy $(\mathrm{P}<0.001)$. Also, there was a positive and significant relationship between safety behavior and self-management with self-efficacy $(\mathrm{P}<0.001)$. In addition, the present study showed that the job stress and safety behavior play a mediator role between self-management and self-efficacy of emergency staff.

Conclusion: In general, considering that job stress, safety behavior and self-management predictive self-efficacy of emergency staff. Individuals with high self-efficacy wait for the desired outcomes of their performance. So by giving stress management training and applying their safety principles and strategies, targeting can create a sense of self-efficacy such that a person can withstand persistent efforts to overcome obstacles. Improving working conditions also plays an effective role in increasing productivity.

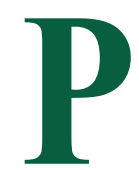

\section{Introduction}

re-Hospital Emergency Medicine (PHEM)

includes all medical assistance (either direct or indirect) performed outside the hospital environment. Emergency medical technicians often face stressful and challenging situations involving injury, unconsciousness or even death. These factors often cause the emergency

\section{* Corresponding Author:}

Reza Noroozi, $P h D$

Address: Department of Management, Tehran Branch, Payame Noor University, Tehran, Iran.

E-mail: rezanorouzi20@pnu.ac.ir 
personnel to suffer from psychological distress. Working in such stressful environments can increase the risk of occupational damage, cardiovascular diseases, mental health disorders, and job burnout [1]. Occupational stress is one of the issues in the field of management and organizational behavior. Organizations pay much attention to the study, control, and reduction of stress in the workplace so that the costs of physical and mental damages of stress are reduced and the productivity of their employees is improved [2]. The causes of stress are divided into organizational and personal areas that directly affect the employees and their job. Job stresses not only cause problems for the individual but also have unfavorable consequences for the organizations. Statistics have showed that stress and its complications result in the loss of hundreds of working days each year, with an average of one million people daily being absent from work due to stress disorders [3]. It has also been reported that $4 \%$ of working hours are lost due to absenteeism resulted from job stress and dissatisfaction [3]. Hospital staff experiences significant job stress because they are the first to be present in emergency situations such as road accidents, natural disasters, or minor injuries and illnesses [1]. Mehdizadeh et al. [3] have showed a negative correlation between job stress and selfefficacy. Kavei et al. [4] have reported that there was no significant difference in general self-efficacy and occupational stress between the two groups.

Self-management is a skill based on self-awareness and self-efficacy. This emotional ability depends on the person's capacity to relieve himself/herself; remove anxiety, depression and common boredom; and delay satisfaction. Self-management is a training process that persuades and guides individuals to achieve a particular behavior and desired outcome. This concept is developed from the theories of self-influence, which emphasizes self-guidance, self-control, and self-management [5].

Studies have explained the cognitive processes leading to personal motivation (spontaneity) and self-management. Bandura [6] has showed that people who expect positions and believe that external outcomes will be sought after their efforts would have stronger self-management skills than those who expect failure and cannot communicate between their efforts and the real-life. In general, self-management is defined as the process of the personal direction of tendencies, behavior, and recognition of individuals toward fulfilling tasks or goals. It is considered as a strong driver of development in all fields since it provides a model for each field. To realize their goals, the individuals should be able to distinguish between their feelings, behaviors, and beliefs; identify their relationships; and become familiar with self-development strategies.
They should consider strategies that can transform their tendencies, behaviors, and cognition [7]. Sassani Moghaddam and Baharololum have reported that there is a positive and significant relationship between self-management and stress in female athlete students [8]. In addition, Ismail Poor and Ebrahimi have concluded that self-management has a significant role in predicting the perceived stress [9].

The term "safety culture" was first formally expressed in 1986 by the IAEA in the Chernobyl incident report and was used to justify the organizational error and individual deficiency that grounded the disaster situation. Many research works have focused on this subject so far [2]. The organizational culture plays the rhythm of doing anything in the realm of safety. In a positive and safe culture, the culture itself creates an atmosphere in which everything relevant to safety is considered important [10]. Safety and health studies have showed that $85-98 \%$ of occupational accidents occurring due to unsafe behaviors are rooted in poor safety culture [11]. These studies have also showed that positive attitudes toward safety that make people look for a safer environment to reduce insecure behaviors and improve their safety status [11]. Safety behaviors include safety precautions and safety participation that are considered as components of safety performance. Safety precautions include activities that the individuals must perform to maintain and improve their personal safety and health. These behaviors include compliance with the standard operating instructions, the use of personal protective equipment and the like. Safety participation includes behaviors that do not directly contribute to the safety of individuals but help to create conditions that enhance the level of safety and health in the organization. These behaviors facilitate the achievement of organization's health and safety goals and programs. Some of these behaviors are participation in voluntary safety activities, helping colleagues in safety matters, and attending safety meetings [12].

Fuchin and Chin have concluded that self-efficacy has a direct impact on the safety behavior of pilots [13]. Self-efficacy is one of the basic concepts of Bandura's theory [6]. It includes individual beliefs about the ability to organize and effectively carry out assignments in a specific area that leads to certain goals [6]. It is based on four sources of successful experiences, substitution experiences, verbal incentives, and emotional and physiological states, which are not informative but are learned through cognitive processing [6]. Occupational self-efficacy is also defined as a person's judgment about his/her ability to carry out the assignment within a given professional and occupational context [14]. It affects and interacts with motivation and behavior, and its high levels increase the participation in occupational assign- 
ments and behaviors [14]. Self-efficacy theory is based on the assumption that people's belief in their abilities and talents has a favorable effect on their actions and is the most important determinant of behavior [6].

Self-efficacy affects the choice of behavior, effort, perseverance, and pursuit of goals and determines how to deal with obstacles and challenges. It plays an important role in psychological adaptation, mental health problems, physical health, as well as strategies for changing self-guided behavior [15]. It is important in stressful occupations and plays a role in preserving and moderating the relationship between the requirements of emotional occupations and mood disorder [16]. Worcesova and Malader have concluded that there is a negative correlation between teachers' self-efficacy and stress [17]. In his research, Chiu has reported that interpersonal stress is the ability to negatively predict social self-efficacy [18]. Employees who believe in their efficiency, struggle better to overcome problems.

Self-efficacy is an important concept that influences the behavior and outcome of the actions. Given the important and influential job of emergency medical staff, empowering individuals to play an effective role and enhance self-efficacy is one of the most important factors in improving their efficiency and performance. Also, there is a lack of research on the indirect impact of occupational stress and safe behavior on emergency medical staff. Thus, the present study aimed to determine the relationship between self-management and self-efficacy with the mediating role of job stress and safe behavior.

\section{Materials and Methods}

The present research is a descriptive correlational study. The study population included all the emergency staff, i.e., the road and urban staff, of Ardebil province in 2017. For sample selection, 5 persons from each of the 40 centers across the province were selected using regular random sampling method. All the participants were then asked to complete the following questionnaires.

Table 1. Mean and standard deviation of the variables

\section{Harris Job Stress Questionnaire (1995)}

The Harris Job Stress Questionnaire questionnaire can provide the most accurate results about the level of job stress in your organization in the shortest time possible. It includes 35 questions on a 5-item Likert scale (never, rarely, sometimes, often, and always), and measures the stress induced by demand, control, peer support, support from supervisors, communication, task, and change. The scores of each item represent the measured value ranging from 1 to 5 , in which 1 is undesirable, and 5 is desirable. In the research of Azad Marzabadi and Gholami Fesharaki, the Cronbach's alpha coefficient was approximately 0.78 , indicating the reliability of this questionnaire [19]. Using the two-half method and the Spearman-Brown formula, the correlation coefficient was measured to be 0.65 for the entire questionnaire.

\section{General Self-efficacy Scale}

The Schwarzer and Grosselm's self-efficacy scale has 10 questions to which the respondent answers on a 4-item Likert scale. The lowest score is 1, and the highest score for each question is score 4 . The overall score in this questionnaire indicates self-efficacy. In a study carried out by Hosseini et al. in Iran, the Cronbach's alpha coefficient for the entire scale was found to be 0.86 [20].

\section{Safety Behavior Questionnaire}

This questionnaire is prepared for assessing the safety behavior with 12 questions in the field of safety precaution and 11 questions in the field of safety participation. It has very good reliability and reliability and also is considered as a native tool for assessing safety behaviors with appropriate accuracy. In this questionnaire, higher scores represent safer behavior. To assess the validity of the questionnaire, 12 health and safety experts were asked to survey the questionnaire [12]. The relevance, simplicity, and clarity of the questions were evaluated to assess its validity using the multi-dimensional scale. The correlation coefficient of this questionnaire between the

\begin{tabular}{ccc}
\hline Variable & Mean & 5.08 \\
\hline Self-management & 24.67 & 6.12 \\
Safety behavior & 52.41 & 6.71 \\
Job stress & 68.55 & 21.72 \\
Self-efficacy & & 4.02 \\
\hline & & IHlealth in \\
Emergencies and |D]isasters [Oluarterly
\end{tabular}


Table 2. Summary of the results of multiple regression equations with self-efficacy criterion variable

\begin{tabular}{ccccc}
\hline Predictor & $\begin{array}{c}\text { Multiple Correlation } \\
\text { Coefficients }\end{array}$ & $\begin{array}{c}\text { Determination } \\
\text { Coefficient }\end{array}$ & F-Statistic & Significance Level \\
\hline $\begin{array}{c}\text { Self-management, Safety behavior, } \\
\text { Job stress }\end{array}$ & 0.533 & 0.28 & 31.26 & 0.001 \\
\hline & & & $\begin{array}{c}\text { 1:lealth in } \\
\text { Emergencies and [Disasters [Oluarterly }\end{array}$ \\
\hline
\end{tabular}

Table 3. Model fitting indices

\begin{tabular}{ccccccc}
\hline Index & $\chi^{2}$ & Sig. & GFI & AGFI & CFI & RMSEA \\
\hline Value & 2.30 & 0.211 & 0.990 & 0.965 & 0.991 & 0.06 \\
\hline & & & & & $\begin{array}{c}\text { Illeath in } \\
\text { Emergencies and [Disasters (Oluarterly }\end{array}$
\end{tabular}

results of the test and re-test is equal to 0.619 . Also, the Cronbach's alpha coefficient of this questionnaire in the study and standardization was 0.766 [12].

\section{Self-Management Strategy Questionnaire}

Self-management was measured by 13 items of behavioral strategies in the self-report questionnaire [21]. Three questions are related to self-targeting strategy, two questions to self-rewarding, three questions to selfpunishment, three questions to self-observation, and two questions to self-guidance. The KMO index is significant for each of the 5 subscales. Also, the Cronbach's alpha coefficient in an internal study was 0.822 [22].

\section{Results}

Of the total 200 participants, the data of only $185 \mathrm{em}$ ployees were analyzed after incomplete questionnaires were removed. Among these participants, 55 subjects had an Associate degree, 105 Bachelor's degree and 25 had Master's degree and higher education levels. The mean age of the participants was $31.11 \pm 5.76$ years, and the mean work experience was $5.33 \pm 4.12$ years. In Table 1 , the mean and standard deviation of the variables and their dimensions are shown.

Before testing the research hypotheses, the role of a predictor of self-management, job stress and safety behavior on self-efficacy was investigated for the purpose of obtaining more information. Multiple correlation coefficients of self-management, job stress and safety behavior with self-efficacy was $\mathrm{R}=533$, indicating a high correlation between these variables and self-efficacy. This correlation coefficient shows that $28 \%$ of the participants' self-efficacy variance is explained by three variables of self-management, job stress, and safety behavior. The role of these three variables in predicting self-efficacy was significant $(\mathrm{P}<0.001)$ (Table 2$)$. To investigate the mediating role of job stress and safety behaviors in the relationship between self-management

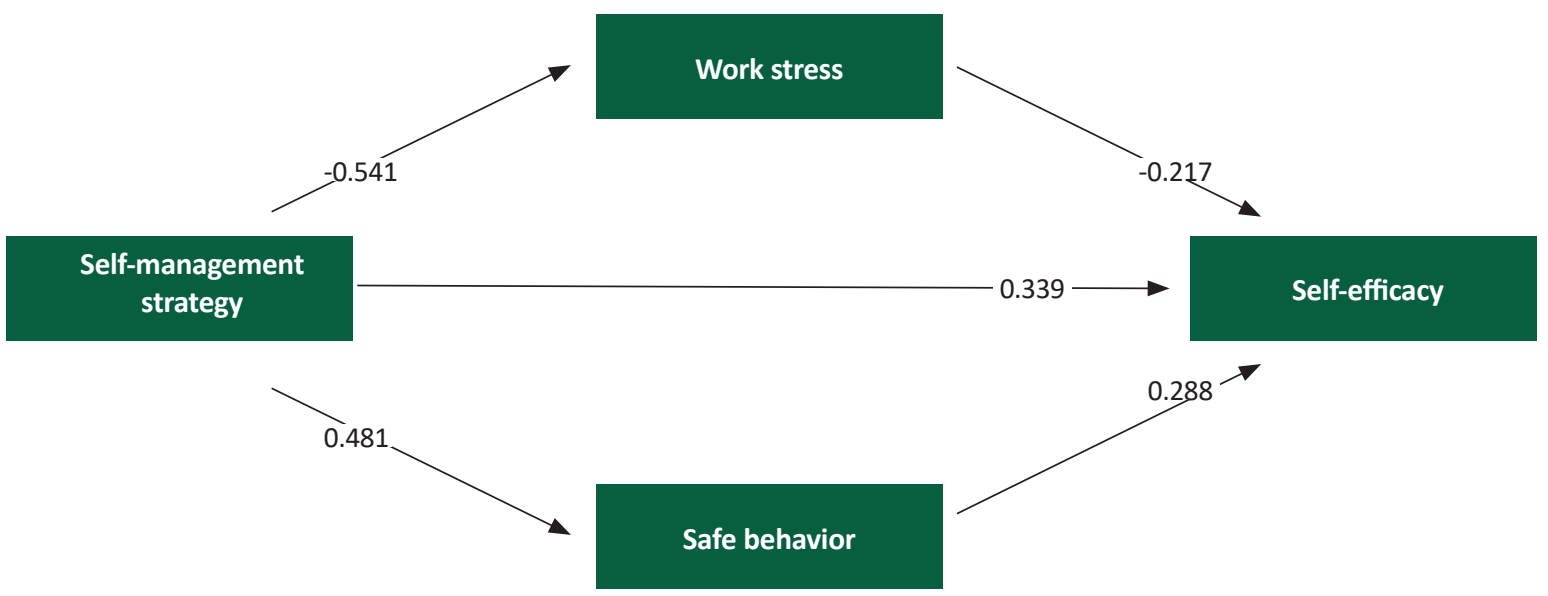

IHealth in

Emergencies and |D] isasters [Oluarterly

Figure 1. The fitted model for the mediating role of job stress and safety behavior in the relationship between self-management strategy and self-efficacy (all path coefficients are significant, $\mathrm{P}<0.001$ ) 
strategy and self-efficacy of emergency staff, path analysis was used.

As shown in Figure 1, all path coefficients were found to be significant $(\mathrm{P}<0.001)$. In other words, self-management strategies directly affect the self-efficacy of emergency staff, and the self-management strategies also have indirect effects on self-efficacy through increasing safe behavior and reducing job stress. The fitting indices of the model are presented in Table 3 to confirm the hypothesized model.

As shown in Table 3, the chi-square value is insignificant $(\mathrm{P}<0.05)$, and all fitting indices of the model have also reached the ideal fit. Goodness of Fit Index (GFI), and Comparative Fit Index (CFI) are above 90\%, which are desirable for fitting the model. The Root Mean Square Error Estimate (RMSEA) was 0.06, which is also considered desirable.

Regarding the fitting indices of the model, it can be said that the assumed model has a desirable fit, and the research hypotheses have been confirmed.

\section{Discussion}

The present study was conducted to investigate the mediating role of job stress and safe behavior in the relationship between self-management strategy and selfefficacy of emergency staff. The study results showed that self-management affects self-efficacy both directly and indirectly through stress and safe behavior.

A significant relationship was found between self-management and occupational stress. This is in accordance with the findings of Ismail Poor and Ebrahimi who concluded that self-management has a significant role in predicting perceived stress [9]. In addition, Sassani Moghadam and Bahrololum also showed a positive and significant relationship between the self-management component and the response to stress [8].

In this study, a negative relationship was observed between job stress and self-efficacy. This result is consistent with the results obtained by Mehdizadeh et al. [3], and Rasova and Mala [17]. Chiu also reported that interpersonal stress is the ability to negatively predict social self-efficacy [18]. We also found that there is a relationship between safe behavior and self-efficacy. This result is consistent with the results of Fochin and Chin who also reported that self-efficacy is directly related to safe behavior [13]. Stressful situations and unsafe behaviors can cause tension and pressure and thereby, distract people and affect their efficacy. The high level of occupational stress will have a negative effect on the efficiency and focus of the staff, and consequently a reduction in productivity. The results also showed that there is a significant relationship between self-management strategy and safe behavior and self-efficacy. Our findings showed that self-management can predict self-efficacy. People with higher self-management have higher goals for themselves, expect better outcomes, and overcome obstacles and problems. Self-efficacy affects the amount of effort that a task requires.

\section{Conclusion}

In conclusion, it can be said that the nature of work in the emergency department unwillingly makes people be in trouble, such that their mental health may be threatened. Thus, it is important to pay attention to the individual and mental capacities by which the individual would be able to withstand the difficult conditions and not suffer damage, and even improve his/her character by experiencing such a problem or crisis situation. Self-efficacy affects the choice of behavior, effort, perseverance, and pursuit of goals, and determines how to deal with obstacles and challenges. Self-efficacy in stressful occupations is essential, and its high levels increase individual participation in occupational assignments and behaviors.

\section{Acknowledgments}

This research did not receive any specific grant from funding agencies in the public, commercial, or not-forprofit sectors. The confidentiality of the information of the participants and the informed consent of all sample individuals were the ethical points of this research. I really appreciate the officials and personnel of the Road and Municipal Emergency department of Ardebil province for their sincere cooperation.

\section{Conflict of Interest}

The authors declared no conflicts of interest.

\section{References}

[1] Moradi Z, Eslami AA, Hasanzadeh A. [Job burnout status among pre-hospital emergency technicians (Persian)]. Iranian Journal of Emergency Medicine. 2015; 2(1):28-32.

[2] Zamanian Z, Azad P, Ghaderi F, Bahrami S, Kouhnavard B. [Investigate the relationship between rate of sound and local 
lighting with occupational stress among dentists in the city of Shiraz (Persian)]. Journal of Health. 2016; 7(1):87-94.

[3] Mehdizadeh P, Pourreza A, Allahverdipour H, Dopeykar $\mathrm{N}$. [Assessing relationship between job stress, self efficacy and coping among teaching hospitals staff in Tabriz University of Medical sciences in 2009 (Persian)]. Journal of Hospital. 2013; 12(1):57-66.

[4] Kavehei T, Ashouri A, Habibi M. [Predicting job satisfaction based on self-efficacy beliefs, teachers' sense of efficacy, job stress and Hierarchy of needs in exceptional children's teachers at lorestan province (Persian)]. Exceptional Education. 2014; 4(126):5-15

[5] Shokri O, Toolabi S, Ghanaei Z, Taghvaei A, Kakabaraei K, Fooladvand KH. [Psychometric study of Self-Efficacy Beliefs Questionnaire (Persian)]. Motaleat-e Amoozesh va Yadgiri. 2010; 3(2):45-61.

[6] Bandura A. Self-efficacy: Toward a unifying theory of behavioral change. Psychological Review; 1977; 84(2):191-215. doi: 10.1037/0033-295x.84.2.191

[7] Karimi Valadani M. [The effect of self-management program on self-management and general health status of hemodialysis patients of the Foundation for Special Diseases (Persian)] [MSc. thesis]. Tehran: University of Social Welfare and Rehabilitation Sciences; 2010.

[8] Sasanimogadam S, Bahrololom H. [Relationship between emotional intelligence with psychological skills in female athletes (Persian)]. Research in Sport Management \& Motor Behavior. 2011; 1(1):27-39.

[9] Esmaeilpour K, Ebrahimi L. [Role of personality and emotional intelligence on perceived stress among employees of motogen company (Persian)]. Modern Psychological Research. 2015; 10(37):25-44.

[10] Roughton JE, Mercurio JJ. Developing an Effective Safety Culture: A Leadership Approach [P. Nasiri, S. Alizade, M. Ahmadlu, Persian trans]. Tehran: Islamic Azad University; 2007.

[11] Jafari MJ, Gharari M, Kalantari S, Omidi L, Ghaffari M, Fardi GRA. [The influence of safety training on improvement in safety climate in construction sites of a firm (Persian)]. Journal of Safety Promotion and Injury Prevention. 2015;2(4):257-64.

[12] Mahdinia M, Arsanqjang S, Sadeghi A, Malakouti J, Karimi A. [Development and validation of a questionnaire for safety behavior assessment (Persian)]. Iran occupational health journal. 2016; 13(2):92-102.

[13] Chen CF, Chen SC. Measuring the effects of Safety Management System practices, morality leadership and selfefficacy on pilots' safety behaviors: Safety motivation as a mediator. Safety Science. 2014; 62:376-85. doi: 10.1016/j. ssci.2013.09.013

[14] Betz NE, Hackett G. The relationship of career-related selfefficacy expectations to perceived career options in college women and men. Journal of Counseling Psychology. 1981; 28(5):399-410. doi: 10.1037/0022-0167.28.5.399

[15] Maddux JE. Self-efficacy: The power of believing you can. In: Lopez SJ, Snyder CR, editors. The Oxford Handbook of Positive Psychology. Oxford: Oxford University Press; 2002.
[16] Jex SM, Bliese PD, Buzzell S, Primeau J. The impact of self-efficacy on stressor-strain relations: coping style as an explanatory mechanism. Journal of Applied Psychology. 2001; 86(3):401-9. PMID: 11419800

[17] Verešová M, Malá D. Stress, proactive coping and self- efficacy of teachers. Procedia - Social and Behavioral Sciences. 2012; 55:294-300. doi: 10.1016/j.sbspro.2012.09.506

[18] Chiu SI. The relationship between life stress and smartphone addiction on Taiwanese university student: A mediation model of learning self-Efficacy and social self-Efficacy. Computers in Human Behavior. 2014; 34:49-57. doi: 10.1016/j.chb.2014.01.024

[19] Azad marzabadi E, Gholami Fesharaki M. [Reliability and validity sssessment for the HSE job stress questionnaire (Persian)]. Journal of Behavioral Sciences. 2011; 4(4):291-97.

[20] Hosseini Dowlatabadi F, Saadat S, Ghasemi Jobaneh R. [Relationship between parenting styles, self- efficacy and attitude to delinquency among high school students (Persian)]. Criminal Law Research. 2013; 4(2):67-88.

[21] Houghton JD, Neck CP. The revised self-leadership questionnaire. Journal of Managerial Psycholog. 2002; 17(8):67291. doi: 10.1108/02683940210450484

[22] Rahimnia F, Eslami G. [The effects of employees' daily self-management on their job engagement: Case of Khorasan Regional Electricity Company (Persian)]. Journal of Management and Development Process. 2015; 28(1):77-100 